CARDIOVASCULAR MEDICINE

\title{
Left atrioventricular plane displacement but not left ventricular ejection fraction is influenced by the degree of aortic stenosis
}

\author{
E Rydberg, P Gudmundsson, L Kennedy, L Erhardt, R Willenheimer
}

Heart 2004;90:1151-1155. doi: 10.1136/hrt.2003.020628

See end of article for authors' affiliations

.....................

Correspondence to: Dr Eric Rydberg, Department of Cardiology, Malmö University Hospital, S-205 02 Malmö, Sweden; erik.rydberg@medforsk. mas.lu.se

Accepted 13 January 2004

\begin{abstract}
Aims: To examine how left atrioventricular plane displacement (AVPD), a widely used measure of left ventricular (LV) function, is related to presence and degree of aortic stenosis.

Methods and results: Cardiac dimensions, LV filling, left AVPD, LV ejection fraction (LVEF), and valve function were assessed by echocardiography/Doppler in 182 patients with various cardiac diseases (mean (SD) age 69 (12) years, 36\% women), 49 consecutive with and 133 consecutive without aortic stenosis. In an analysis of covariance, neither left AVPD nor LVEF was independently correlated with the presence of aortic stenosis. However, looking separately at patients with aortic stenosis, left AVPD ( $p=0.03$ ) but not LVEF correlated independently with degree of aortic stenosis in multiple linear regression analysis. In patients with aortic stenosis, an abnormal left AVPD had $94 \%$ sensitivity and $90 \%$ negative predictive value with regard to severe aortic stenosis, compared with $56 \%$ and $62 \%$, respectively, for LVEF.

Conclusion: In patients with cardiac disease, neither left AVPD nor LVEF correlated independently with presence of aortic stenosis. However, in patients with aortic stenosis, left AVPD but not LVEF correlated with the degree of aortic valve obstruction and left AVPD but not LVEF had high sensitivity and negative predictive value with regard to severe aortic stenosis. Compared with LVEF, left AVPD is an earlier and more sensitive marker of LV haemodynamic load in patients with aortic stenosis.
\end{abstract}

M easurement of left atrioventricular plane displacement (AVPD) by echocardiography is a clinically useful method for the assessment of left ventricular (LV) function. It is uncomplicated, rapid, and highly reproducible. ${ }^{1-8}$ It is particularly useful since left AVPD is readily measured also in echocardiograms with poor image quality. ${ }^{67}$ Left AVPD is a strong prognostic marker in patients with heart failure, stable coronary artery disease, and acute myocardial infarction. ${ }^{67-11}$ It has been shown to correlate well with LV ejection fraction (LVEF) calculated by two dimensional echocardiography, radionuclide ventriculography, and contrast cineangiography. ${ }^{1-4}$ However, left AVPD and LVEF are different expressions of LV function. It has been suggested that left AVPD is caused predominantly by contraction of subendocardial, longitudinal myocardial fibres, whereas LVEF depends mainly on contractility of subepicardial, circumferential myocardial fibres. ${ }^{12}{ }^{13}$ We have shown that left AVPD, besides being related to LV systolic function, is related to LV diastolic performance, which most likely explains some of the discrepancies between left AVPD and LVEF. ${ }^{8}{ }^{14}$

In patients with aortic stenosis, the haemodynamic load with increased afterload causes a rise in LV wall stress and myocardial hypertrophy develops in response to this. Increased wall thickness and decreased cavity size oppose the increased wall stress. The LV hypertrophy initially leads to diastolic dysfunction and, later on, to systolic dysfunction. ${ }^{15}$ In about two thirds of patients with aortic stenosis LVEF is normal, whereas in one third it is depressed. ${ }^{16}$ In the late stages, LV systolic function can be reduced as a result of myocardial fibrosis. ${ }^{17}$ However, before that, increased wall stress can cause LV systolic dysfunction. An inverse linear relation between end systolic wall stress and circumferential fibre shortening has been shown and several studies have shown a close inverse relation between LVEF and wall stress induced by aortic stenosis. ${ }^{17-23}$

The sequence of cardiac decompensation begins with ventricular dilatation, which further raises wall stress, leading to increased LV hypertrophy and probably reduced blood flow to the hypertrophied myocardium, in turn leading to ischaemia with reduced LVEF. The subendocardial fibres are more sensitive than the epicardial fibres to ischaemia. Since left AVPD mainly reflects subendocardial performance and LVEF mainly epicardial fibre performance, ${ }^{12}{ }^{13}$ and since left AVPD is indicative of both systolic and diastolic function, ${ }^{8}{ }^{14}$ left AVPD may be an earlier and more sensitive marker than LVEF of LV haemodynamic load in patients with aortic stenosis.

The aim of the present study was to examine how left AVPD is related to the presence and degree of aortic stenosis.

\section{METHODS \\ Patients}

We enrolled 49 consecutive patients with and 133 consecutive patients without aortic stenosis during 14 and four months, respectively, referred to our echocardiography laboratory for a diagnostic examination at the department of cardiology in Malmö University Hospital, with a primary catchment area of 250000 inhabitants. The reasons for referral were mainly assessment of LV and valve function in patients with coronary artery disease, heart failure, and valve disease, but no referral reason excluded the patient. Since atrial fibrillation has been shown to decrease left AVPD ${ }^{11}{ }^{24-26}$ and since LV diastolic performance cannot be adequately assessed by

Abbreviations: AVPD, atrioventricular plane displacement; $E: A$, ratio of early to atrial peak velocity of diastolic transmitral flow; LV, left ventricular; LVEF, left ventricular ejection fraction 
Doppler in patients with atrial fibrillation, only patients in sinus rhythm were included. The definition of coronary artery disease was prior or current myocardial infarction, angina verified with exercise test, stress echocardiography, radionuclide examination, or coronary angiography, and prior percutaneous transluminal coronary angioplasty or coronary bypass grafting.

\section{Echocardiographic examination}

Two dimensional echocardiography and Doppler examinations were performed by either of two investigators with a Hewlett Packard (Andover, Massachusetts, USA) Sonos 2000 or 2500 echocardiography system and a $2.5 \mathrm{MHz}$ transducer. Pulsed, continuous, and colour flow Doppler examinations were performed with the same transducers. Parasternal and apical views were obtained with the patient in a left lateral recumbent position. Measurements were acquired during silent respiration or end expiratory apnoea.

Left AVPD was determined by two dimensionally guided M mode in the four and two chamber views, as described previously. ${ }^{6-8}$ The regional AVPD (mm) was the distance covered by the atrioventricular plane between the position most remote from the apex (corresponding to the onset of contraction) and the location closest to the apex (corresponding to the end of contraction, including any postejection shortening) - that is, the full extent of the displacement. It was measured in the septal, lateral, posterior, and anterior regions and was calculated from an average of two to four heartbeats. The mean of the AVPD in the four regions was calculated. The mean interobserver variability in our laboratory between two investigators examining each patient immediately after one another was $4.8 \%$ (AVPD difference range $0-1.2 \mathrm{~mm}$ ) in a series of 53 consecutive patients with a mean left AVPD of $7.8 \mathrm{~mm}$ (range 3.3-15.5 mm). ${ }^{6}$ The intraobserver variability of the determination of left AVPD was mean $2.0 \%$ (range $0-6 \%$ ), corresponding to $0.23 \mathrm{~mm}$ (range $0-0.95 \mathrm{~mm}$ ), in 39 randomly examined patients with a mean left AVPD of $11.2 \mathrm{~mm}$ (range 5.6-17.5 mm). ${ }^{27}$

LVEF was determined by visual quantification and was evaluated independently of left AVPD, based on regional and global wall motion assessment. ${ }^{14}$ This is a routine method in our laboratory and measurements against examinations with previously determined LVEF are regularly calibrated to avoid drift in the assessments. Two experienced investigators always determined the LVEF. When there was a discrepancy between the investigators regarding the LVEF the examination was re-evaluated until they agreed. The variability between the investigators evaluating LVEF in the present study was tested in a series of 26 patients with a mean LVEF of 0.48 (range 0.17-0.70). The mean difference of the LVEF determinations was 0.02 (range $0.00-0.07$ ), corresponding to a $5.6 \%$ mean difference (range $0-19 \%$ ).

LV diastolic filling was assessed by transmitral and pulmonary venous pulsed Doppler echocardiography. The transmitral Doppler examination was performed as described previously ${ }^{28}$ by determining the ratio of early to atrial peak velocity of diastolic transmitral flow (E:A). ${ }^{29}$ Pulmonary venous flow was examined in the apical four chamber view with the cursor placed parallel to the flow and the sample volume 0.5 to $1.0 \mathrm{~cm}$ into the right upper pulmonary vein. The relation between the systolic (S) and diastolic (D) component of the inflow was assessed. LV diastolic performance was graded on the basis of S:D and E:A. It was considered to be normal (grade 1) when E:A was $>0.75$ and $\leqslant 1.0$ or when E:A was $>1.0$ combined with $\mathrm{S}: \mathrm{D}>1.0$. Diastolic filling was considered mildly impaired (grade 2) when E:A was $\leqslant 0.75$ and moderately to severely impaired (grade 3) when E:A was $>1.0$ and S:D $\leqslant 1.0$ (pseudonormalised or abnormally high E:A). The lower E:A limit (0.75) of the normal diastolic filling interval was chosen based on the study by Mantero and colleagues ${ }^{30}$ showing that the E:A in healthy patients of an age similar to the mean age of our patients was $1.0(0.2)$. In healthy middle aged people the maximum flow velocity of the systolic pulmonary venous component is higher than that of the diastolic inflow. ${ }^{31-34}$ Therefore $\mathrm{S} \leqslant \mathrm{D}$ was considered to be abnormal.

The degree of aortic valve obstruction was evaluated by calculating the velocity-time integral ratio of the LV outflow tract systolic pulsed Doppler flow to the systolic continuous Doppler aortic flow from traces of the respective signals in the apical five chamber view. ${ }^{35}$ Significant aortic stenosis was defined as a velocity-time integral ratio of $<0.40$. This corresponds roughly to a valve area of 1.2$1.3 \mathrm{~cm}^{2}$ in a person with an average body surface area. Severe aortic stenosis was defined as a velocity-time integral ratio of $<0.23$.

Cardiac dimensions were measured in the parasternal long axis view in two dimensional mode. ${ }^{36} \mathrm{LV}$ mass was calculated from two dimensionally obtained measures, according to the recommendations of the American Society of Echocardiography, with a necropsy validated formula. ${ }^{36}{ }^{37}$

Grading of valvar regurgitation was based on colour Doppler signal area, continuous Doppler signal density, and continuous Doppler pressure half time (aortic regurgitation only) in the parasternal and apical views. The grades were none (0), mild (1), mild to moderate (2), moderate (3), moderate to severe (4), and severe (5). With this method, interobserver and intraobserver variability was a maximum of one grade in our laboratory.

\section{Statistical analysis}

The $t$ test was used to test differences between two groups. For assessment of correlations between variables, univariate and multivariate linear regression analyses, covariance analysis, and the Spearman rank correlation test were used. Data are expressed as mean (SD). Two tailed $p$ values $<0.05$ were considered significant. Variables that in univariate regression analysis were significantly correlated $(\mathrm{p}<0.05)$ with left AVPD and LVEF were considered for inclusion in multivariate linear regression analysis. However, interventricular septum diameter was excluded, since it is highly correlated with LV posterior wall diameter and since LV posterior wall diameter is a better marker of LV mass than interventricular septum diameter. We wanted to explore the relation between LV diameter and left AVPD and, since LV mass is a function of LV wall thickness and LV diameter, LV mass was also excluded. E:A was excluded, since it was part of the "LV diastolic function" variable.

\section{Ethics}

The study is approved by the ethics committee of Lund University.

\section{RESULTS}

The mean (SD) age of all patients was 69 (12) years; 65 (36\%) were women. Table 1 shows the most important variables analysed in the present study for patients with and patients without aortic stenosis. Twenty four $(49 \%)$ of the patients with aortic stenosis and $110(83 \%)$ of the patients without aortic stenosis had coronary artery disease $(p<0.0001)$. The presence of coronary artery disease did not correlate with left AVPD or LVEF in any patient group. Among patients with aortic stenosis, 26 (53\%) had angina pectoris, 25 (51\%) had congestive heart failure, $8(16 \%)$ had a history of syncope, and $8(16 \%)$ had a history of dizziness. Left AVPD, but not LVEF, was significantly lower in patients with aortic stenosis than in those without. 
Table 1 Age, body surface area, and echocardiographic and Doppler variables in patients with and without aortic stenosis

\begin{tabular}{|c|c|c|c|c|c|}
\hline \multirow[b]{2}{*}{ Variable } & \multicolumn{2}{|c|}{ Aortic stenosis $(n=49)$} & \multicolumn{2}{|c|}{ No aortic stenosis ( $n=133$ ) } & \multirow[b]{2}{*}{ p Value } \\
\hline & Mean (SD) & Range & Mean (SD) & Range & \\
\hline Age (years) & $77(8)$ & $53-90$ & $67(12)$ & $35-90$ & $<0.0001$ \\
\hline Body surface $\left(\mathrm{m}^{2}\right)$ & $1.77(0.18)$ & $1.39-2.09$ & $1.92(0.20)$ & $1.51-2.42$ & $<0.0001$ \\
\hline IVSDd $/ \mathrm{m}^{2}(\mathrm{~mm})$ & $7.4(1.9)$ & $4.1-13.3$ & $5.6(1.0)$ & $3.6-9.0$ & $<0.0001$ \\
\hline LVPWDd/m² $(\mathrm{mm})$ & $7.2(1.5)$ & $4.9-12.2$ & $5.9(0.8)$ & $4.1-8.1$ & $<0.0001$ \\
\hline $\mathrm{LVIDd} / \mathrm{m}^{2}(\mathrm{~mm})$ & $27.3(3.4)$ & $18.4-34.2$ & $26.6(4.2)$ & $13.6-39.8$ & 0.285 \\
\hline $\mathrm{LVM} / \mathrm{m}^{2}(\mathrm{~g})$ & $137(43)$ & $76-266$ & $112(32)$ & $48-234$ & $<0.0001$ \\
\hline $\mathrm{LAs} / \mathrm{m}^{2}(\mathrm{~mm})$ & $24.7(3.4)$ & $18.5-34.2$ & $22.1(2.9)$ & $15.6-32.5$ & $<0.0001$ \\
\hline AVPD $(\mathrm{mm})$ & $9.2(2.3)$ & $4.8-15.5$ & $10.7(2.8)$ & $4.4-17.0$ & 0.001 \\
\hline LVEF (\%) & $50(12)$ & $20-70$ & $52(14)$ & $15-70$ & 0.542 \\
\hline $\mathrm{E}: \mathrm{A}$ & $1.08(0.89)$ & $0.15-3.60$ & $1.08(0.60)$ & $0.30-4.50$ & 0.967 \\
\hline Diastolic performance (grade 1-3) & $2.1(0.57)$ & $1-3$ & $1.9(0.67)$ & $1-3$ & 0.026 \\
\hline MR & $1.9(1.0)$ & $0-5.0$ & $1.5(0.9)$ & $0-4.0$ & 0.013 \\
\hline VTI ratio & $0.25(0.07)$ & $0.12-0.39$ & NA & NA & NA \\
\hline Aortic $V_{\max }(\mathrm{m} / \mathrm{s})$ & $3.8(1.0)$ & $2.1-6.6$ & NA & NA & NA \\
\hline
\end{tabular}

\section{Left AVPD}

Tables 2, 3, and 4 show variables that correlated significantly with left AVPD in univariate analysis in the entire patient group, in patients with aortic stenosis, and in patients without aortic stenosis. In the entire patient group, AVPD correlated significantly with the presence of aortic stenosis. In patients with aortic stenosis, the velocity-time integral ratio correlated significantly with AVPD. This correlation was closer in patients without coronary artery disease $(r=0.58$, $\mathrm{p}=0.004)$ than in patients with coronary artery disease $(r=0.39, \mathrm{p}=0.07)$. Variables that correlated independently with left AVPD in multivariate analysis in the entire patient group were age $(p<0.0001)$, LVPWD $(p<0.001)$, LV size $(p<0.0001)$, and LV diastolic filling ( $p=0.0001)$. Among patients with aortic stenosis, the variables that qualified for the multivariate linear regression analysis explained $52 \%$ of the left AVPD variability. Age $(\mathrm{p}=0.041)$, velocity-time integral ratio $(\mathrm{p}=0.026)$, and LV diastolic filling $(p=0.023)$ correlated independently with left AVPD. The variables that qualified for the multivariate linear regression analysis in patients without aortic stenosis explained $48 \%$ of the left AVPD variability. Age $(p=0.002)$, LV size $(p=0.0001)$, LVPWD $(p=0.029)$, and LV diastolic filling $(p=0.002)$ correlated independently with left AVPD. Among patients with aortic stenosis, an abnormal left AVPD-defined as $<11.0 \mathrm{~mm}$, which corresponds to an LVEF $<55 \%{ }^{1}$-had a negative predictive value of $90 \%$ and a sensitivity of $94 \%$ with regard to severe aortic stenosis (table 5). Of the 17 patients with depressed left

Table 2 Variables that significantly correlated with AVPD in univariate linear regression analyses in the entire patient group

\begin{tabular}{lrr}
\hline Variable & $\boldsymbol{r}$ & p Value \\
\hline Age (years) & -0.52 & $<0.0001$ \\
Body surface $\left(\mathrm{m}^{2}\right)$ & 0.32 & $<0.0001$ \\
$\mathrm{IVSDd} / \mathrm{m}^{2}(\mathrm{~mm})$ & -0.30 & $<0.0001$ \\
$\mathrm{LVM} / \mathrm{m}^{2}$ & -0.46 & $<0.0001$ \\
$\mathrm{LVPWDd} / \mathrm{m}^{2}(\mathrm{~mm})$ & -0.41 & $<0.0001$ \\
$\mathrm{LVIDd} / \mathrm{m}^{2}(\mathrm{~mm})$ & -0.47 & $<0.0001$ \\
$\mathrm{LAs} / \mathrm{m}^{2}(\mathrm{~mm})$ & -0.45 & $<0.0001$ \\
Presence/absence of aortic stenosis & -0.24 & 0.0012 \\
Diastolic performance (grade 1-3) & $-0.36^{*}$ & $<0.0001^{*}$ \\
MR & -0.38 & $<0.0001$ \\
\hline *Spearman rank correlation test. & & \\
\hline
\end{tabular}

AVPD despite non-severe aortic stenosis, 11 had coronary artery disease.

\section{LVEF}

In univariate analysis in the entire patient group, age $(\mathrm{p}<0.0001)$, LV size $(\mathrm{p}<0.0001)$, left atrial diameter $(\mathrm{p}<0.0001)$, mitral regurgitation $(\mathrm{p}<0.0001)$, LV diastolic filling $(\mathrm{p}<0.0001)$, but not presence of aortic stenosis $(p=0.54)$ correlated with LVEF. In multivariate analysis, LV size $(\mathrm{p}<0.0001)$ and LV diastolic filling $(\mathrm{p}=0.0004)$ remained independent correlates. Table 6 shows significant correlates of LVEF in univariate analysis among patients with aortic stenosis. The velocity-time integral ratio did not correlate with LVEF, neither in patients without coronary artery disease $(r=0.40, \mathrm{p}=0.06)$ nor in patients with coronary artery disease $(r=0.23, \mathrm{p}=0.30)$. In multivariate regression analysis, explaining $47 \%$ of the LVEF variability in patients with aortic stenosis, only LV size $(\mathrm{p}=0.012)$ correlated independently with LVEF. Among patients with aortic stenosis, an abnormal LVEF $(<55 \%)$ had a negative predictive value of $62 \%$ and a sensitivity of $56 \%$ with regard to severe aortic stenosis (table 5). Of the 13 patients with depressed LVEF despite non-severe aortic stenosis, 10 had coronary artery disease.

\section{DISCUSSION}

Aortic stenosis imposes increased afterload and increased wall stress on the LV. The increased afterload is offset by the development of concentric LV hypertrophy by the process of sarcomere replication. ${ }^{38}$ Compensated aortic stenosis is

Table 3 Variables that correlated significantly with AVPD in univariate linear regression analyses in patients with aortic stenosis

\begin{tabular}{lrl}
\hline Variable & \multicolumn{1}{l}{$\boldsymbol{r}$} & $\mathbf{p ~ V a l u e ~}$ \\
\hline Age (years) & -0.45 & 0.001 \\
Body surface $\left(\mathrm{m}^{2}\right)$ & 0.31 & 0.030 \\
$\mathrm{LVPWDd} / \mathrm{m}^{2}(\mathrm{~mm})$ & -0.39 & 0.006 \\
$\mathrm{LVM} / \mathrm{m}^{2}$ & -0.32 & 0.024 \\
$\mathrm{LVIDd} / \mathrm{m}^{2}(\mathrm{~mm})$ & -0.32 & 0.028 \\
Diastolic performance (grade 1-3) & $-0.37^{*}$ & $0.0009^{*}$ \\
VTI ratio & 0.43 & 0.003 \\
VTI ratio (in patients with VTI ratio <0.23) & 0.20 & 0.055 \\
VTI ratio (in patients with VTI ratio $\geqslant 0.23)$ & 0.12 & 0.54 \\
MR & -0.28 & 0.049 \\
\hline \multirow{2}{*}{ *Spearman rank correlation test. } & \\
\end{tabular}


Table 4 Variables significantly correlated with AVPD in univariate linear regression analyses in patients without aortic stenosis

\begin{tabular}{lcc}
\hline Variable & $\boldsymbol{r}$ & $\mathbf{p ~ V a l u e ~}$ \\
\hline Age (years) & -0.48 & $<0.0001$ \\
Body surface $\left(\mathrm{m}^{2}\right)$ & 0.25 & 0.004 \\
$\mathrm{IVSDd} / \mathrm{m}^{2}(\mathrm{~mm})$ & -0.20 & 0.019 \\
$\mathrm{LVM} / \mathrm{m}^{2}$ & -0.48 & $<0.0001$ \\
$\mathrm{LVPWDd} / \mathrm{m}^{2}(\mathrm{~mm})$ & -0.37 & $<0.0001$ \\
$\mathrm{LVIDd} / \mathrm{m}^{2}(\mathrm{~mm})$ & -0.50 & $<0.0001$ \\
$\mathrm{LAs} / \mathrm{m}^{2}(\mathrm{~mm})$ & -0.50 & $<0.0001$ \\
Diastolic performance (grade 1-3) & $-0.44^{*}$ & $<0.0001^{*}$ \\
MR & -0.38 & $<0.0001$ \\
\hline \multirow{2}{*}{ *Spearman rank correlation test. } & & \\
\hline
\end{tabular}

characterised by concentric hypertrophy and a normal or supranormal LVEF. With longstanding hypertrophy LV contractility may be depressed somewhat, but LVEF is relatively preserved by the Frank-Starling mechanism. LVEF is maintained at the expense of higher $\mathrm{LV}$ end diastolic pressure and volume. When the aortic stenosis reaches a critical degree, the LV cannot recruit additional preload because the maximum sarcomere length is reached and the hypertrophied stiff ventricle resists further filling. ${ }^{18}$ The LVEF and stroke volume are now directly related to the afterload or wall stress, so with further progression of the aortic stenosis LVEF and stroke volume fall in proportion to the increase in wall stress.

Thus, LVEF is not depressed until in rather advanced stages of aortic stenosis. We hypothesised that left AVPD would be an earlier and more sensitive marker than LVEF of LV haemodynamic load among patients with aortic stenosis. Indeed, as opposed to LVEF, left AVPD correlated significantly with the presence of aortic stenosis in univariate analysis, although left AVPD and presence of aortic stenosis were not independently correlated in multivariate analysis. Furthermore, left AVPD but not LVEF was significantly reduced among patients with aortic stenosis compared with patients without aortic stenosis. Moreover, in patients with aortic stenosis, left AVPD but not LVEF was significantly correlated with the degree of aortic valve obstruction. This was especially evident in patients without coronary artery disease, further suggesting a relation between left AVPD and degree of aortic stenosis. An abnormal left AVPD had high sensitivity and negative predictive value with regard to severe aortic stenosis, whereas an abnormal LVEF did not. Thus, left AVPD was depressed if aortic stenosis was severe and a normal left AVPD precluded severe aortic stenosis with a high probability. This was not the case for LVEF. Consequently, compared with LVEF, left AVPD seems to be an earlier and

Table 5 Analysis of sensitivity, specificity, and predictive values for AVPD and LVEF with regard to severe aortic stenosis (VTI ratio $<0.23$ ) in patients with aortic stenosis

\begin{tabular}{lll}
\hline & AVPD $<11.0 \mathrm{~mm}$ & AVPD $\geqslant \mathbf{1 1 . 0} \mathbf{m m}$ \\
\hline VTI ratio $<0.23$ & 17 & 1 \\
VTI ratio $\geqslant 0.23$ & 17 & 9 \\
\hline & LVEF $<\mathbf{5 5 \%}$ & LVEF $\geqslant \mathbf{5 5 \%}$ \\
\hline VTI ratio $<0.23$ & 10 & 8 \\
VTI ratio $\geqslant 0.23$ & 13 & 13 \\
\hline
\end{tabular}

For AVPD: sensitivity (17/18) 94\%; specificity (9/26) 35\%; positive predictive value (17/34) $50 \%$; negative predictive value $(9 / 10) 90 \%$. For LVEF: sensitivity (10/18) 56\%; specificity (13/26) $50 \%$; positive predictive value $(10 / 23) 43 \%$; negative predictive value $(13 / 21) 62 \%$.
Table 6 Variables significantly correlated with LVEF in univariate linear regression analyses in patients with aortic stenosis

\begin{tabular}{llc}
\hline Variable & $\boldsymbol{r}$ & $\mathrm{p}$ Value \\
\hline Age (years) & -0.44 & 0.0016 \\
$\mathrm{LVIDd} / \mathrm{m}^{2}(\mathrm{~mm})$ & -0.54 & $<0.0001$ \\
Diastolic performance (grade 1-3) & $-0.52^{*}$ & $0.0003^{*}$ \\
MR & -0.51 & 0.0002 \\
\hline *Spearman rank correlation test. & & \\
\hline
\end{tabular}

more sensitive marker of LV haemodynamic load among patients with aortic stenosis.

Among patients with aortic stenosis, both left AVPD and LVEF had poor specificity and positive predictive value with regard to severe aortic stenosis. This was mainly because some patients with coronary artery disease had depressed left AVPD and LVEF without having severe aortic stenosis.

A possible explanation for the result of the present study may be that left AVPD is more sensitive to ischaemia caused by increased wall stress because left AVPD reflects subendocardial fibre function. The result may also be explained by the relation between LV diastolic performance and left AVPD, as opposed to LVEF, since diastolic dysfunction occurs before systolic dysfunction in aortic stenosis.

Prior studies have shown that left AVPD is decreased in patients with LV hypertrophy, ${ }^{39}{ }^{40}$ which was also the case in the present study. However, our results indicate that the decrease of left AVPD in relation to the degree of valve obstruction is independent of LV hypertrophy, suggesting a direct relation with the increased intraventricular pressure resulting from aortic stenosis.

\section{Conclusion}

This study shows that left AVPD, as opposed to LVEF, correlated significantly with the presence of aortic stenosis in univariate analysis, although not independently in multivariate analysis. In patients with aortic stenosis, left AVPD correlated independently with the degree of aortic valve obstruction, whereas LVEF did not. This was especially evident among patients without coronary artery disease. An abnormal left AVPD, but not LVEF, also had high sensitivity and negative predictive value with regard to severe aortic stenosis: Patients with severe aortic stenosis are very likely to have an abnormal left AVPD, whereas a normal left AVPD precludes severe aortic stenosis with high probability. Our results indicate that, compared with LVEF, left AVPD is an earlier and more sensitive marker of LV haemodynamic load in patients with aortic stenosis. Future research will have to examine whether depressed left AVPD in patients with aortic stenosis is an ominous prognostic sign indicating that the aortic valve should be replaced.

\section{ACKNOWLEDGEMENTS}

This study was supported by a grant from the Swedish Heart and Lung Foundation.

\section{Authors' affiliations}

E Rydberg, P Gudmundsson, L Kennedy, L Erhardt, R Willenheimer, Department of Cardiology, Malmö University Hospital, Lund University, Malmö, Sweden

\section{REFERENCES}

1 Alam M, Höglund C. Serial echocardiographic studies following thrombolytic treatment in myocardial infarction with special reference to the atrioventricular valve plane displacement. Clin Cardiol 1992;15:30-6. 
2 Alam M, Höglund C, Thorstrand C. Longitudinal systolic shortening of the left ventricle: an echocardiographic study in subjects with and without preserved global function. Clin Physiol 1992;12:443-52.

3 Alam M, Höglund C, Thorstrand C, et al. Atrioventricular plane displacement in severe congestive heart failure following dilated cardiomyopathy or myocardial infarction. J Int Med 1990;228:569-75.

4 Alam M, Höglund C, Thorstrand C, et al. Haemodynamic significance of the atrioventricular plane displacement in patients with coronary artery disease. Eur Heart J 1992; 13:194-200.

5 Wandt B, Boiö L, Wranne B. Influence of body size and age on mitral ring motion. Clin Physiol 1997; 17:635-46.

6 Willenheimer R, Cline C, Erhardt L, et al. Left ventricular atrioventricular plane displacement: an echocardiographic technique for rapid assessment of prognosis in heart failure. Heart 1997:78:230-6.

7 Willenheimer R, Erhardt L, Cline C, et al. Prognostic significance of changes in left ventricular systolic function in elderly patients with congestive heart failure. Coron Artery Dis 1997;8:711-7.

8 Willenheimer R, Israelsson B, Cline C, et al. Left atrioventricular plane displacement is related to both systolic and diastolic left ventricular performance in patients with chronic heart failure. Eur Heart $J$ 1999;20:612-8

9 Brand B, Rydberg E, Ericsson G, et al. Prognostication and risk stratification by assessment of left atrioventricular plane displacement in patients with myocardial infarction. Int J Cardiol 2002:83:35-41.

10 Rydberg E, Willenheimer R, Erhardt L. Atrioventricular plane displacement determined by echocardiography: a clinically useful, independent predictor of mortality in patients with stable coronary artery disease. J Intern Med 2003;254:479-85.

11 Rydberg E, Arlbrandt M, Gudmundsson P, et al. Left atrioventricular plane displacement predicts cardiac mortality in patients with chronic atrial fibrillation. Int J Cardiol 2003;91:1-7.

12 Lundbäck S. Cardiac pumping and the function of the ventricular septum. Acta Physiol Scand 1986;550(suppl):1-101.

13 Henein MY, Priestley K, Davarashvili T, et al. Early changes in left ventricular subendocardial function after successful coronary angioplasty. Br Heart J 1993;69:501-6.

14 Rydberg E, Willenheimer R, Brandt B, et al. Left ventricular diastolic filling is related to the atrioventricular plane displacement in patients with coronary artery disease. Scand Cardiovasc J 2001;35:30-4.

15 Lund O, Flo C, Jensen FT, et al. Left ventricular systolic and diastolic function in aortic stenosis. Eur Heart J 1997; 18:1977-87.

16 Krayenbuehl HP, Hess OM, Ritter ES, et al. Left ventricular systolic function in aortic stenosis. Eur Heart J 1988;9(suppl E):19-23.

17 Krayenbuehl HP, Hess OM, Monrad ES, et al. Left ventricular myocardial structure in aortic valve disease before, intermediate, and late after aortic valve replacement. Circulation 1989;79:744-55.

18 Ross J Jr. Afterload mismatch in aortic and mitral valve disease: implications for surgical therapy. J Am Coll Cardiol 1985;5:811-26.

19 Colan SD, Borow KM, Neumann A. Left ventricular end-systolic wall stressvelocity of fiber shortening relation: a load-independent index of myocardial contractility. J Am Coll Cardiol 1984;4:715-24.

20 Gunther S, Grossman W. Determinants of ventricular function in pressure overload hypertrophy in man. Circulation 1979;59:679-88.

21 DePace NL, Ren J-F, Iskandrian AS, et al. Correlation of echocardiographic wall stress and left ventricular pressure and function in aortic stenosis. Circulation 1983;67:854-9.
22 Carabella BA, Green LH, Grossman W, et al. Hemodynamic determinants of prognosis of aortic valve replacement in critical aortic stenosis and advanced congestive heart failure. Circulation 1980;56:22-30.

23 Dineen $\mathrm{E}$, Brent $\mathrm{BN}$. Aortic valve stenosis: comparison of patients with to those without chronic congestive heart failure. Am J Cardiol 1986;57:419-22.

24 Alam M, Thorstrand C. Left ventricular function in patients with atrial fibrillation before and after cardioversion. Am J Cardiol 1992;69:694-6.

25 Emilsson K, Wandt B. The relation between ejection fraction and mitral annulus motion before and after direct-current electrical cardioversion. Clin Physiol 2000;20:218-24.

26 Emilsson K, Wandt B. The relation between mitral annulus motion and left ventricular ejection fraction in atrial fibrillation. Clin Physiol 2000;20:44-9.

27 Willenheimer R. Assessment of left ventricular dysfunction and remodeling by determination of atrioventricular plane displacement and simplified echocardiography. Scand Cardiovasc J 1998;32(suppl):48.

28 Pearson AC, Nelson J, Kanter J, et al. Effect of sample volume location on pulsed Doppler-echocardiography evaluation of left ventricular filling. Am J Card lmaging 1988;2:40-6.

29 Oh JK, Appleton CP. Hatle LK, et al. The noninvasive assessment of left ventricular diastolic function with two-dimensional and Doppler echocardiography. J Am Soc Echocardiogr 1997; 10:246-70.

30 Mantero A, Gentile F, Gualtierotti C, et al. Left ventricular diastolic parameter in 288 normal subjects from 20 to 80 years old. Eur Heart J 1995; 16:94-105.

31 Nishimura RA Abel MD, Hatle LK, et al. Assessment of diastolic function of the heart: background and current applications of Doppler echocardiography. Part II. Clinical studies. Mayo Clin Proc 1989;64:181-204.

32 Rossvoll O, Hatle LK. Pulmonary venous flow velocity recorded by transthoracic Doppler ultrasound: relation to left ventricular diastolic pressures. J Am Coll Cardiol 1993:21:1687-96.

33 Mulvagh S, Quinones MA, Kleiman NS, et al. Estimation of left ventricular end-diastolic pressure from Doppler transmitral flow velocity in cardiac patients independent of systolic performance. J Am Coll Cardiol 1992;20:112-9.

34 Appleton CP, Hatle LK, Popp RL. Relation of transmitral flow velocity patterns to left ventricular diastolic function: new insights from a combined hemodynamic and Doppler echocardiographic study. J Am Coll Cardiol 1988; 12:426-40.

35 Otto C, Pearlman A, Comess K, et al. Determination of the stenotic aortic valve area in adults using Doppler echocardiography. J Am Coll Cardiol 1986;7:509-17.

36 Schiller NB, Shah PM, Crawford M, et al. Recommendations for quantitation of the left ventricle by two-dimensional echocardiography. American Society of Echocardiography committee on standards, subcommittee on quantitation of two-dimensional echocardiograms. J Am Soc Echocardiogr 1989;2:358-67.

37 Devereux RB, Alonso DR, Lutas EM, et al. Echocardiographic assessment of left ventricular hypertrophy: comparison to necropsy findings. Am J Cardiol 1986;57:450-8.

38 Carabella BA. The relationship of the left ventricular geometry and hypertrophy to left ventricular function in valvular heart disease. $J$ Heart Valve Dis 1995:4(suppl II):S132-9.

39 Wandt B, Bojö L, Tolagen K, et al. Echocardiographic assessment of left ventricular ejection fraction in left ventricular hypertrophy. Heart 1999;81:192-8.

40 Emilsson K, Wandt B. The relation between mitral annulus motion and ejection fraction changes with age and heart size. Clin Physiol 2000;20:38-43. 